\title{
Guest editorial: Web technologies and applications
}

\author{
Quan Z. Sheng • Jing He • Guoren Wang • \\ Christian S. Jensen
}

Received: 23 January 2014 /

Accepted: 29 January 2014 / Published online: 1 March 2014

(C) Springer Science+Business Media New York 2014

The relentless growth in Web functionality and broadband access has enabled a new wave of innovations that are transforming the way people and organizations interact, communicate, and collaborate. This special issue of the WWW Journal collects five articles, which are extensions of the best research papers presented at APWeb 2012 (the 14th Asia-Pacific Web Conference, Kunming, China). These papers went through several rounds of rigorous review by the guest editors and invited reviewers.

The first paper, by Peng and Wang, "Member Promotion in Social Networks via Skyline," focuses on an interesting research topic in social networks, namely member promotion that aims at discovering potential stars. The authors propose a skyline-based approach for member promotion in social networks and systematically study the problem in two different settings: unequal-weighted social networks and equal-weighted social networks.

The paper, by Hu et al., "A Unified Framework for Semi-Supervised PU Learning," proposes a new framework that integrates a range of existing semi-supervised PU learning algorithms to better tackle the problem of learning from positive yet unlabeled data. The authors also develop an automatic KL-divergence learning method capable of utilizing the knowledge of unlabeled data distributions. The experimental results demonstrate the better performance of their approach.

Q. Z. Sheng $(\bowtie)$

School of Computer Science, The University of Adelaide, Adelaide, SA 5005, Australia

e-mail: qsheng@cs.adelaide.edu.au

J. He

College of Engineering and Science, Victoria University, Victoria, VIC 8001, Australia

G. Wang

School of Information Science and Engineering, Northeastern University, Liaoning, 110819, China

C. S. Jensen

Department of Computer Science, Aalborg University, 9220, Aalborg Øst, Denmark 
The next two papers focus on the topic of outlier detection. The paper, by Shaikh and Kitagawa, "Efficient Distance-based Outlier Detection on Uncertain Datasets of Gaussian Distribution," focuses on uncertain-data management and more specifically proposes a novel approach that enables approximate but more efficient cell-based outlier detection. The paper, by Zhang et al., "Outlier Detection from Large Distributed Databases," concentrates on multiple large distributed databases. The authors present a system called DISTROD (DISTRibuted Outlier Detector) that is able to effectively detect global outliers from distributed databases using a number of optimization and performance enhancing strategies.

Finally, the paper by Huang et al., "Online Mining Abnormal Period Patterns from Multiple Medical Sensor Data Streams," focuses on the very practical challenge of detecting abnormalities in frequently changing medical sensor data streams. The authors develop a MAPPMPS (Mining Abnormal Period Patterns from Multiple Physiological Streams) method and demonstrate its efficiency and effectiveness using a massive real-world data set.

The papers included in this special issue cover several important topics and present some of the key directions in the vibrant and rapidly expanding area of World Wide Web research and related research areas. We hope that these papers provide the community with a better understanding of the current directions and will serve to inspire continued research.

Acknowledgements We would like to thank the referees who provided very useful and thoughtful feedback to the authors. We also express our gratitude to the Editor-in-Chief, Professor Yanchun Zhang, for his kind support, advice, and encouragements throughout the preparation of this special issue. 